\title{
Current Concepts of Using Pigs as a Source for Beta-Cell Replacement Therapy of Type 1 Diabetes
}

\author{
Nikolai Klymiuk ${ }^{1,2}$ - Barbara Ludwig ${ }^{3,4}$. Jochen Seissler ${ }^{5}$ - Bruno Reichart ${ }^{6}$. \\ Eckhard Wolf ${ }^{1,2,4}$
}

Published online: 10 May 2016

(C) Springer International Publishing AG 2016

\begin{abstract}
The global prevalence of insulin-dependent diabetes mellitus is rapidly increasing. In spite of major improvements in insulin treatment regimens and diabetes technology (e.g., artificial pancreas devices), glucose control remains problematic in a substantial proportion of diabetic patients. Those patients may benefit from beta-cell replacement therapies. Allotransplantation of pancreas or isolated pancreatic islets is limited by the small number of organ donors. Thus, alternative sources of beta-cells are being developed and tested. These include endocrine progenitor cells or mature beta-cells derived from pluripotent human stem cells and attempts to derive human pancreas tissue in animal hosts by interspecific chimeric complementation experiments. Xenotransplantation of porcine islets is a realistic alternative option. Immune rejection of xenoislets can be prevented by immunosuppression of the recipient or by encapsulation of the islets in microdevices or macrodevices. Using precise and efficient genetic engineering of donor pigs, immune-protected xenoislets with improved functionality can be generated.
\end{abstract}

This article is part of the Topical Collection on Preclinical Animal Models in Regenerative Medicine

Nikolai Klymiuk

n.klymiuk@gen.vetmed.uni-muenchen.de

Eckhard Wolf

ewolf@1mu.de

Barbara Ludwig

barbara.ludwig@uniklinikum-dresden.de

Jochen Seissler

jochen.seissler@med.uni-muenchen.de

Bruno Reichart

bruno.reichart@med.uni-muenchen.de

1 Gene Center and Department of Veterinary Sciences, LMU Munich, Feodor-Lynen-Str. 25, 81377 Munich, Germany
Keywords Diabetes · Islet of Langerhans · Pig · Xenotransplantation $\cdot$ Genetic engineering $\cdot$ Encapsulation

\section{Introduction}

Current data from the International Diabetes Federation indicates that 387 million people worldwide suffer from diabetes, and this is predicted to increase to 592 million by 2035 (https://www.idf.org/diabetesatlas). About $10 \%$ of these subjects suffer from type 1 diabetes, which involves immune-mediated destruction of the insulin-producing pancreatic beta-cells and thus requires exogenous insulin replacement. Although the incidence and prevalence of type 1 diabetes can vary substantially even between neighboring regions, the global incidence of type 1 diabetes has been increasing for several decades (reviewed in [1]). The absolute number of insulin-dependent patients is huge: in 2012, the number of type 1 diabetic children and adults in the USA was estimated

2 Center for Innovative Medical Models (CiMM), LMU Munich, Hackerstr. 27, 85764 Oberschleißheim, Germany

3 Department of Medicine III, University Hospital Carl Gustav Carus, Technical University of Dresden, Fetscherstr. 74, 01307 Dresden, Germany

4 Paul Langerhans Institute Dresden of Helmholtz Centre Munich at University Clinic Carl Gustav Carus of TU Dresden and DZD-German Centre for Diabetes Research, Neuherberg, Germany

5 Diabetes Center, Medizinische Klinik und Poliklinik IV, LMU Munich, Ziemssenstr. 1, 80335 Munich, Germany

6 Walter Brendel Center of Experimental Medicine, LMU Munich, Marchioninistr. 15, 81377 Munich, Germany 
at 1.25 million (http://www.diabetes.org/diabetes-basics/ statistics/), and-based on recently summarized incidence data [2] - the worldwide prevalence is in the range of tens of millions. It is likely that different therapeutic options will be required for patient groups with different subtypes of insulindependent diabetes.

Classical insulin treatment regimens involve multiple daily injections (MDIs) of long-acting insulin providing basal insulin and rapid-acting insulin administered before meals, doseadjusted to pre-meal blood glucose, carbohydrate intake, and anticipated physical activity (reviewed in [3]). Continuous subcutaneous insulin infusion (CSII) is an alternative option. No systematic differences in glycated hemoglobin (A1C) levels or severe hypoglycemia rates were observed between these two forms of intensive insulin therapy in children and adult patients [4].

Over the last decade, remarkable advances in diabetes technology have been made (reviewed in [5]). The latest insulin pump technology and sensor-augmented insulin pumps, preferably with an automated low-glucose suspend feature, have entered diabetes therapy in patients with unstable glycemic control, i.e. two or more episodes per year of severe hypoglycemia or one episode associated with impaired awareness of hypoglycemia, extreme glycemic lability, or major fear and maladaptive behavior [6]. Without doubt, novel diabetes technology has already had a major impact on diabetes care and patients' quality of life. However, closed-loop systems and fully artificial pancreas devices still face several technical hurdles that prohibit their widespread application and preclude the goal of physiological metabolic control [7]. These are (1) insufficient accuracy and stability of continuous glucose monitoring systems and unsuitable measuring sites; (2) lack of "ultra-rapid" insulin with the appropriate pharmacokinetics, i.e., fast onset, rapid peak, short duration of action; (3) insufficient algorithms including features for exercise, stress, sleep, and illness; (4) suboptimal user interface (device-user, devicesensor-pump-controller, device-cloud); and (5) inappropriate sites for device application (nonphysiological route of insulin delivery). Numerous study groups are involved in developing advanced artificial pancreas systems, but implementation is hampered by safety issues and tremendous economic hurdles.

For patients with recurrent life-threatening hypoglycemia, pancreas or islet transplantation is considered as ultimate therapeutic option (reviewed in [6, 8]). Both can normalize glucose homeostasis and prevent macrovascular and microvascular complications of diabetes [1]. Pancreas transplants are mostly performed as simultaneous pancreas-kidney (SPK) transplants in patients with end-stage kidney failure due to diabetic microangiopathy. One-year insulin independence rates of $77 \%$ and 5 -year graft survival of $72 \%$ were reported (reviewed in [9]). While pancreas transplantation is usually performed in younger patients, islet transplantation is also possible in older patients since the procedure is less invasive and islets can be isolated from pancreata that would not be suitable for whole organ transplantation. The major advantage of islet cell transplantation over whole organ transplantation is constituted by the transplantation itself as a minimally invasive procedure associated with very few complications [10]. Data from the Collaborative Islet Transplant Registry (CITR) for the years 2007 to 2010 revealed 66, 55, and $44 \%$ insulinindependent patients at 1,2 , and 3 years after intraportal islet transplantation [11], and more recently, even $50 \%$ insulinindependence at 5 years after transplantation has been reported (reviewed in [9, 12]). Pancreas and islet transplants are considered as complementary approaches and the only evidence-based therapeutic options to overcome problematic hypoglycemia in type 1 diabetes [6]. However, requirement of livelong immunosuppression and the critical lack of human donor organs are major limitations that urgently call for alternative options including porcine tissue.

\section{Beta-Cells Derived from Stem Cells}

Different strategies to generate new beta-cells are currently being discussed (reviewed in [13]). One is the directed differentiation of pluripotent stem cells through the various stages of beta-cell development into endocrine progenitors or functional beta-cells; the other involves reprogramming of terminally differentiated cell types, such as acinar or alpha-cells, in vivo to generate beta-cells. Very recently, reprogrammed stomach tissue has been found to be a source of functional beta-cells in a mouse model [14•].

Beta-cells derived in vitro from either embryonic stem cells (ESCs) or induced pluripotent stem cells (iPSCs) of allogenic or autologous origin are discussed as a possible alternative to islet allotransplantation. In vitro derivation of beta-cells from stem cells is obviously not easy and takes considerable time (ESC 3 weeks, iPSC 3-6 months). When successful, insulin synthesis may be inconsistent and secretion dynamics do not resemble normal patterns. De-differentiation to the original state (ESC, iPSC) is possible and risks teratoma formation $[15,16 \bullet, 17,18]$. Allogeneic cells would be an abundant source but are immunogenic. This drawback and the need for immunosuppression could be overcome by establishing haplobanks, but this will be difficult to organize and require international cooperation to provide tissues or cells with the best possible MHC match for an individual patient. Stem cell-derived human beta-cells would still be prone to autoimmune attack, so tolerance induction would be needed. An alternative is encapsulation. Recently, long-term (6 months) glycemic control in streptozotocin (STZ)-induced diabetic, immune-competent C57BL/6 mice has been observed after intraperitoneal transplantation of human ESC-derived beta-cells microencapsulated in 1.5-mm spheres of triazole thiomorpholine dioxide (TMTD) alginate [19•]. Use of this chemically modified 
polymer prevents fibrosis of implanted microcapsules [20•]. After demonstrating the preclinical safety and efficacy of human ESC-derived pancreatic progenitor cells transplanted in a retrievable Encaptra macroencapsulation device subcutaneously into SCID/beige mice [21], the Californian company Viacyte Inc. has recently commenced a phase I/II clinical study of this product (http://www.jdrf.ca/jdrf-cctn/currentlyrecruiting/viacyte-encapsulation-trial/). So far, no long-term efficacy studies with stem cell-derived beta-cells in diabetic large animal models or patients have been completed.

\section{Attempts to Develop Human Pancreas in Animal Hosts}

Proof of concept for developing an entirely allogeneic or xenogeneic pancreas in intraspecific or interspecific chimeras was first provided by the group of Hiromitsu Nakauchi (Tokyo University) [22]. They used mouse blastocysts with defective copies of the pancreatic and duodenal homeobox 1 $(P d x l)$ gene, which is essential for pancreas development. Embryos and fetuses developing from these blastocysts have an empty pancreas niche, and apancreatic offspring die shortly after birth from severe hyperglycemia. This lethal phenotype could be rescued by injecting ESC or iPSC from $P d x 1$-intact mouse strains into the $P d x l$-defective blastocysts. The resulting chimeras had a pancreas derived from injected pluripotent stem cells. Injection of rat iPSC into $P d x l$-defective mouse blastocysts resulted in interspecific chimeras with a mostly rat-derived pancreas, demonstrating that intraspecific and interspecific blastocyst complementation with pluripotent stem cells can form an entire organ in a host engineered to have a free organogenic niche.

As a first step toward generation of a human pancreas in an animal host, Matsunari and coworkers [23•] generated pancreatogenesis-disabled transgenic pigs that express Hairy enhancer split-1 (Hes1) under PDX1 promoter control. Since no fully functional porcine pluripotent stem cells are available, blastocyst complementation was performed with embryonic blastomeres expressing the fluorescent marker KusabiraOrange (K-O). Resulting chimeric fetuses and offspring had pancreata derived from the K-O-labeled cells. The availability of specific organogenesis-impaired pig hosts opens the possibility of targeted organ generation from human pluripotent stem cells, but whether this is feasible between phylogenetically distant species remains to be seen. Masaki et al. [24] developed an assay to test the ability of pluripotent stem cells to form interspecies chimeras, and found that human iPSC failed to integrate into the epiblast of mouse egg-cylinder stage embryos. This they ascribed to different gastrulation mechanisms and incompatibility of ligands or adhesion molecules and proposed the use of host embryos from more closely related species.
In addition to biological difficulties, such use of human pluripotent stem cells raises ethical issues, for example the possibility of unanticipated human contributions to neurons or even germ cells [25]. A possible solution would be stem cells with limited differentiation potential. Kobayashi et al. [26] have demonstrated that inducible expression of Mixlike protein 1 (Mixl1) limits the differentiation potential of mouse pluripotent stem cells to derivatives of the endodermal germ layer. Such cells could still form pancreas, but not neurons or other ectoderm or mesoderm derivatives. Different concepts of interspecies chimeric complementation using totipotent, pluripotent, or multipotent stem cells from one species in a different host species to generate tissues mainly derived from the stem cell donor species have been extensively discussed in a recent review [27•].

An alternative for human tissue formation is the generation of vascularized complex organ buds from stem cell-derived tissue-specific progenitor cells, endothelial cells, and mesenchymal stem cells (MSCs). Takebe et al. [28] demonstrated that murine beta-cells (MIN6), human umbilical vein endothelial cells (HUVECs), and MSCs formed condensed tissue buds when cocultured on a hydrogel substrate of appropriate stiffness. After several days in culture, these cell condensates developed into large, mechanically stable, self-organizing 3D tissues, which - after transplantation to mice-formed isletlike structures and a functional microvascular network which was connected to the recipient's circulation. Importantly, after transplantation under the kidney capsule, these in vitro derived pancreatic buds were able to restore normal weight gain and blood glucose levels of diabetic mice. While such pancreatic progenitor tissue buds could also be formed from differentiated human iPSC derivatives, the size of a mouse as host for further differentiation into pancreatic progenitor tissue is not sufficient to generate tissue grafts for human patients. Pigs could be more suitable interim hosts for this approach, as demonstrated by the development of de novo kidneys of reasonable size from metanephroi transplanted into the omentum of pigs ([29]; comment in [30]). Potential immune rejection of human tissue buds in pig hosts could be overcome by using genetically immuno-compromised or immune-deficient pigs [31].

In summary, the concept to generate human tissues in animal hosts via interspecies chimeric complementation is interesting, but at present not mature for clinical application [27•].

\section{Xenotransplantation of Porcine Islets}

A number of reasons argue for the use of pigs as islets donor species for the treatment of insulin-dependent patients [32]. These include (i) the similarity of porcine and human insulin and the fact that porcine insulin has been used for decades in diabetes therapy; (ii) the high fecundity of pigs; (iii) the 
availability of efficient and precise techniques for genetic modification; (iv) the possibility of maintaining pigs under designated pathogen-free (DPF) conditions; and (v) less socio-cultural and ethical concerns compared to research involving nonhuman primates (NHPs) or dogs.

Islets from adult donor pigs or neonatal islet cell clusters (NICCs) have been most widely used. In addition, experiments with fetal pig islets and also with pancreatic primordia from pig embryos have been performed. The pros and cons of these sources have recently been summarized [33].

Islets from adult pigs are characteristically mature in structure and fully functional as demonstrated by their glucosestimulated insulin secretion in vitro and their ability to immediately restore normoglycemia after transplantation into diabetic recipients. Retired female breeders of large size are usually favored as islet donors as they appear to be most consistent in islet yield and quality. Nevertheless, the isolation of adult pig islets remains technically challenging and expensive.

In contrast, the isolation of NICCs is well established and standardized. The procedure is more robust and less costly than islet isolation from adult donor pigs. Since NICCs have the capacity to proliferate, their cell mass can increase after transplantation. However, freshly isolated NICCs are not fully functional and require maturation in vitro or-after transplantation - in vivo until beta-cells secrete enough insulin to be therapeutically effective. The optimal transplantation site and efficient vascularization of NICCs after transplantation are additional issues to be solved [33].

In general, there are three strategies to facilitate engraftment and prolonged function of porcine xenoislets:

- Free pig islets are transplanted with immunosuppression and tolerance inducing protocols

- Pig islets are encapsulated, in which case immunosuppression will not be needed

- Genetically modified islets are used under the protection of refined immunosuppression with reduced toxicity

\section{Preclinical Studies with Free Islets and Immunosuppression in Nonhuman Primates}

Remarkable progress has been made in restoring glucose control by transplanting wild-type (WT) porcine islets into diabetic NHP with immunosuppression. In 2006, two publications reported long-term maintenance of normoglycemia after intraportal transplantation of porcine NICCs (about $6.2 \times 10^{6}$ beta-cells $/ \mathrm{kg}$ ) in pancreatectomized rhesus macaques [34] or adult pig islets (25,000 islet equivalents/kg; cultured for $48 \mathrm{~h})$ in STZ-induced diabetic cynomolgus macaques [35]. For this success, massive immunosuppression of the recipients, including CD154-specific monoclonal antibodies, was necessary that would not be acceptable in a clinical setting.
Thompson and coworkers [36] treated STZ-induced diabetic rhesus macaques with intraportal infusions of $\sim 50,000$ islet equivalents $/ \mathrm{kg}$ WT neonatal porcine islets. Maintenance immunosuppression of cohort 1 included belatacept and mycophenolate mofetil (MMF) plus induction with basiliximab and lymphocyte function-associated antigen-1 (LFA-1) blockade. Cohort 2 had additional tacrolimus induction. Cohort 3 received alefacept instead of basiliximab and a more intense LFA-1 blockade. In contrast to cohort 1, cohorts 2 and 3 achieved sustained insulin-independent normoglycemia (median rejection-free survivals 60 and 111 days) demonstrating that sparing the CD40/CD154 pathway blockade is possible.

In a more recent study, Shin and coworkers [37•] used adult WT porcine islets for intraportal treatment of STZ-induced diabetic rhesus macaques. Immunosuppressive induction included cobra venom factor (CVF), anti-thymocyte globulin (ATG), anti-TNF $\alpha$ antibody (adalimumab), and maintenance treatment was performed with anti-CD154 monoclonal antibody and low-dose sirolimus. The islet grafts survived and were able to control glucose homeostasis for $>603,512$, 303,180 , and 167 days.

In spite of these promising results with WT pig islets, immediate translation into clinical application is hampered by the large number of islets required and by the need for immune suppression. In particular, anti-CD154 monoclonal antibody is clinically not applicable because it is thrombogenic [38].

\section{Microencapsulation and Macroencapsulation of Wild-Type Porcine Islets}

Encapsulation of pancreatic islets within mainly alginatebased microcapsules or macrocapsules with various physical configurations may allow protection of the islet graft without the need for immunosuppressive agents and safe extension of the donor pool to animal tissue [39]. A large number of transplantation studies with encapsulated islets of different origin have been performed in different rodent models, and several in large animal models or even human clinical trials (reviewed in [40]). While in rodents many different studies with encapsulated islets showed promising results, most of these approaches failed or revealed more disparate results when tested in large animal models or human patients.

Xenotransplantation studies of porcine islets into NHP were mostly done using the microencapsulation approach. Dufrane et al. [41] showed that microencapsulated porcine islets transplanted under the kidney capsule of nondiabetic cynomolgus macaques can survive and function for 6 months. However, the potential of microencapsulated porcine islets to restore normoglycemia in diabetic NHP models was limited (reviewed in [32]). This may - at least in part—be due to species-specific differences in insulin/C-peptide levels required to maintain sustained normoglycemia (C-peptide levels of 0.47 to $3.14 \mathrm{nmol} / 1$ in NHP compared to 0.11 and 
$0.32 \mathrm{nmol} / \mathrm{l}$ in pigs). After transplantation of pig islets into diabetic NHP, recipients with porcine C-peptide levels within the normal range for nondiabetic pigs showed improved glucose homeostasis, but only in some cases insulin independence [42]. This limitation is, however, specific for widely used NHP models and would be less critical in human patients as their blood glucose and C-peptide levels are more similar to pig than to NHP [42].

The main issues to be resolved in islet xenotransplantation are associated with graft oxygenation, sufficient immune protection, inflammatory response, material biocompatibility, and optimal transplantation. The long-term success of encapsulation strategies may be hampered by pericapsular fibrosis and limited survival of the encapsulated islets, especially after intraperitoneal implantation [43]. Recent evidence indicates that these problems may be partially overcome by choosing encapsulation materials with improved biocompatibility $[20 \bullet, 44]$.

At the IPITA-IXA-CTS Conference 2015 in Melbourne, the New Zealand Company LCT in cooperation with Diatranz Otsuka Limited (DOL), Japan (http://www. dolglobal.com/) presented the results of two clinical phase I/IIa trials involving treatment of type 1 diabetic patients with alginate/ornithine microencapsulated islets performed in New Zealand $[n=14 ; 5000$ and 20,000 islet equivalents (IEQs) [45]] and Argentina ( $n=8$; between 5000 and 10,000 IEQ, given in two doses).

Summarizing the two studies, the following conclusions were reported (Shinichi Matsumoto, Otsuka Pharmaceutical Factory Inc., personal communication):

- The number of hypoglycemic attacks decreased in the treated patients, and levels of glycated hemoglobin (A1C) decreased to less than $7 \%$.

- Porcine insulin levels were not measured and the efficacy was classified as "not stable."

- Improvements, such as better encapsulation techniques and anti-inflammatory treatment, are required.

- There was no transmission of PERV or other microorganisms [46].

Since microcapsules with islets are too large $(0.5-1.0 \mathrm{~mm})$ for intraportal transplantation and have diffusional limitations, attempts to immune-protect pig islets using a thin layer of photopolymerizable polyethylene glycol (PEG) have been published already two decades ago [47]. Recently, this conformal coating approach was refined to achieve highthroughput encapsulation of individual mouse islets into nanoliter droplets of optimized PEG gel, resulting in a thin (tens of micrometers) continuous layer of hydrogel. Conformal coating did not impair the ability of the islets to restore normoglycemia in chemically diabetic syngeneic mice [48•]. However, to our knowledge, no efficacy studies of conformal coated xenoislets in diabetic large animal models have been published.

In contrast, macroencapsulated porcine islets were shown to restore glucose homeostasis in diabetic NHP models.

Pierre Gianello's group (Catholic University of Louvain, Belgium) reported on a small trial with diabetic NHP treated with macrodevices (alginate patches incorporating porcine islets) that were able to control hyperglycemia for up to 6 months [49]. Although no fibrosis or complement deposition was observed on explanted macrodevices, IgG anti-pig antibodies, mainly against the Gal- $\alpha 1,3-\mathrm{Gal}(\alpha \mathrm{Gal})$ epitope, were found in the NHP recipients after explantation.

In the macroencapsulation device developed by the company Beta- $\mathrm{O}_{2}$ Technologies (Rosh-Haayin, Israel), pancreatic islets are embedded in a multilayer immune-protective membrane of alginates and a polytetrafluoroethylene (PTFE) membrane which prevents the access of immunoglobulins while allowing for sufficient supply of oxygen from a central chamber in the device for optimal function of the islets [50]. Oxygen (ambient air) is supplied via two subcutaneous ports $[51 \cdot, 52]$. After the concept of islet macroencapsulation had been successfully proven in different transplant models in large animals (biocompatibility, efficacy, immune-protection) [52] and in a first trial of macroencapsulated allogeneic islet transplantation in man [51•], a study of porcine islet xenotransplantation in diabetic NHP was initiated. The first promising results were reported at the IPITA-IXA-CTS Conference 2015 in Melbourne [53].

\section{Genetic Modification of Islets Donor Pigs}

The goal of genetic modification is to generate improved donor pigs of pancreatic islets which (i) are protected against the different mechanisms of xenogeneic immune rejection; (ii) exhibit improved survival during isolation and culture, and after transplantation; (iii) are readily revascularized after transplantation; (iv) have improved structural and functional properties compared to WT islets; and (v) have reduced risk to spread infectious agents such as porcine endogenous retroviruses (PERVs). During the past two decades, the spectrum of genetic engineering techniques for pigs has expanded rapidly, facilitating efficient and targeted modifications of islet donor pigs [54]. The combination of genetic modifications depends on several factors, including the type of islets (adult, neonatal, fetal) and the transplant site (intraportal, intraperitoneal, intramuscular, under the kidney capsule, etc.).

Intraportal delivery of xenoislets, the main application route in islet allotransplantation [6], may trigger the instant blood-mediated inflammatory reaction (IBMIR), which causes significant islet destruction through activation of complement and coagulation and infiltration by innate immune cells, in particular if NICCs expressing high levels of the xeno-antigen $\alpha \mathrm{Gal}$ are used [55]. Therefore, islets from 
alpha-1,3-galactosyltransferase (GGTA1) deficient (GTKO) pigs lacking $\alpha \mathrm{Gal}$ and/or expressing human complement regulatory proteins have been tested in NHP. GTKO did not influence the outcome of intraportally transplanted adult pig islets, presumably due to the reduction of $\alpha \mathrm{Gal}$ epitopes during postnatal islet development [56]. Expression of hCD46 had no effect on islet loss in the early post-transplant period, but was beneficial for long-term survival of the islets [57]. In STZ-induced diabetic rhesus monkeys with immunosuppression, GTKO was shown to be advantageous for survival and engraftment of NICCs, which express high levels of $\alpha \mathrm{Gal}$ [58]. In combination with transgenic expression of hCD55 and hCD59, GTKO NICCs clearly attenuated IBMIR after intraportal transplantation into nondiabetic baboons [59]. Data from an elegant "dual islet transplant model," which allows comparison of different islet preparations within the same recipient, showed that in the absence of immunosuppression, a robust inflammatory response may precede IBMIR, masking the beneficial effect of GTKO observed in previous studies $\left[60^{\bullet}\right]$. The authors suggested porcine monocyte chemotactic protein $1 /$ chemokine (C-C motif) ligand 2 (MCP1/CCL2) produced by xenogeneic islets as a contributing factor.

Several pro-inflammatory signals released by islets have been identified, with CCL2 being the chemokine that has been payed most attention to (reviewed in [61]). Specifically, CCL2 released from human islets has been shown to impair their engraftment [62] and its blocking has been demonstrated to facilitate permanent survival of murine islet allografts [63]. CCL2 does not only stimulate attraction and activation of innate immune cells, but acts in an autocrine or paracrine manner to further increase the pro-inflammatory state of the islets, e.g., by stimulating the expression of tissue factor (TF) on the surface of islet cells [64]. TF is an important player in IBMIR, stimulating the extrinsic pathway of the coagulation cascade [65]. Since disruption of the $\mathrm{Ccl} 2$ gene in mice does not affect their life span and fertility [66], CCL2-deficient pigs should be viable as well. Thus, inactivation of the CCL2 gene along with insertion of an overexpression cassette for the prominent TF inhibitor TFPI [67] would be a reasonable strategy to reduce both, pro-inflammatory as well as pro-coagulant signals from islet xeno-grafts.

So far, mainly islets with genetic modifications designed to combat complement mediated processes such as IBMIR have been tested in nonhuman primates. Other strategies to prevent immune rejection of xenoislets have been tested in mouse models or in vitro systems.

T-cell-mediated rejection is considered as the major barrier for long-term survival of islet xenografts [68]. We generated transgenic pigs expressing the T-cell costimulation blocking molecule LEA29Y under the control of the porcine INS promoter. After transplantation under the kidney capsule of diabetic NOD-SCID Il2 $\mathrm{rg}^{-/-}$(NSG) mice, LEA29Y expressing
NICCs restored glucose control and were- in contrast to WT NICCs - not rejected by transplanted human blood mononuclear cells (PBMCs). Importantly, only very low levels of LEA29Y were detectable in the circulation of mice grafted with transgenic islets, supporting the concept of local immune modulation by LEA29Y [69•]. These findings were recently extended in diabetic NSG mice grafted with human CD34+ hematopoietic stem cells, where LEA29Y transgenic islets survived and maintained glucose control for more than 6 months (Lelia Wolf-van Buerck and Jochen Seissler, unpublished data). In addition, intramuscular transplantation of NICCs into NSG mice was established [70] to test the effect of LEA29Y at this alternative transplantation site in future studies. First primate transplantation experiments revealed that expression of LEA29Y alone may not be sufficient to promote engraftment upon intramuscular injection of NICCs into nondiabetic marmosets; however, these experiments did not give any evidence for transmission of PERV and porcine cytomegalovirus (PCMV) [71].

A complementary strategy to blocking the CD28-CD80/ CD86 costimulatory pathway is overexpression of the negative costimulatory molecule PDL1 in the xenograft [72]. Transplantation of pig cells overexpressing human PDL1 induced diminished cellular and antibody responses in vivo in a rat model [73]. Expression of PDL1 in xenoislets may thus prevent their T-cell-mediated destruction.

T-cell-mediated islet xenograft rejection involves an interferon-gamma-dependent recruitment of macrophages and natural killer (NK) cells [74]. Vascular endothelial cells from transgenic pigs expressing HLA-E/beta2-microglobulin were effectively protected against human NK cell-mediated cytotoxicity, depending on the level of CD94/NKG2A expression on the NK cells [75]. A protective effect of HLA-E/beta2microglobulin transgene expression in islets remains to be shown.

Other genetic modifications of donor pigs with putative beneficial effects on the outcome of islet xenotransplantation include the removal of additional xenoantigens, such as Neu5Gc (knockout of the cytidine monophosphate-Nacetylneuraminic acid hydroxylase gene $C M A H$ ) and $\operatorname{Sd}(a)$, (knockout of the beta-1, 4-N-acetyl-galactosaminyl transferase 2 gene B4GALNT2) (reviewed in [76]). Preliminary in vitro studies indicated that elimination of Neu5Gc in addition to $\alpha$ Gal may reduce early pig islet loss from IBMIR [77]. In addition, transgenic expression of inhibitors of coagulation and/or inflammation, like tissue factor pathway inhibitor (TFPI), human CD39, human thrombomodulin and human endothelial protein $\mathrm{C}$ receptor, and of cytoprotective molecules like heme oxygenase 1 and A20 has been proposed (reviewed in [78]). A recent study showed the expression of multiple transgenes (porcine CTLA4-Ig, TFPI, human CD39) driven by an insulin promoter on a GTKO/human CD46 transgenic background did not disturb beta-cell function of the 
Fig. 1 Aims of genetic modifications of islets donor pigs. The relevance for free vs. encapsulated islet transplantation is indicated by arrows.

Established or suggested genetic modifications to achieve these aims are detailed in the text

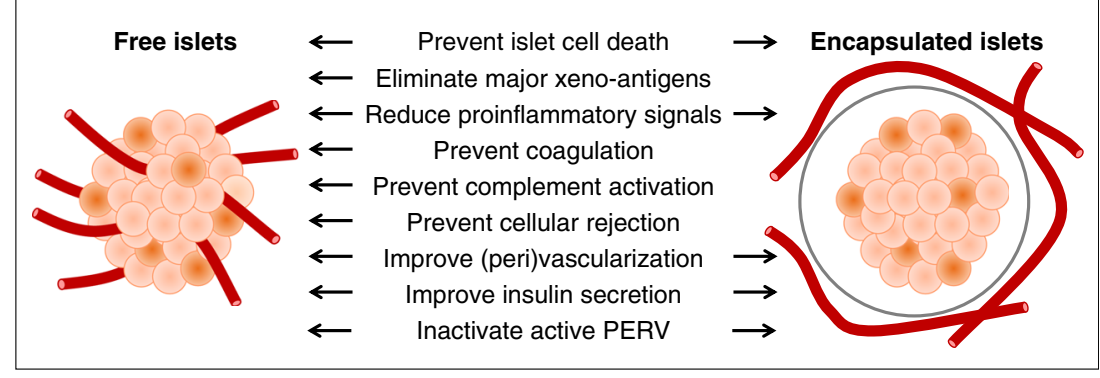

genetically multimodified pigs [79]. Intraportal transplantation experiments of adult pig islets with three, four, or five genetic modifications into diabetic NHP revealed a reduction in early graft loss, but no improvement of long-term outcomes compared historical experiments with human CD46 transgenic or WT islets [80].

A large proportion of pancreatic islets are lost due to apoptosis during isolation, culture, and transplantation. Adenoviral transduction of human islets with X-linked inhibitor of apoptosis protein (XIAP), which blocks caspases 3, 7, and 9, dramatically reduced the number of human islets required for reversing hyperglycemia in diabetic immunedeficient mice [81]. Genetic modification of donor pigs facilitates permanent expression of XIAP in pancreatic islets, which should also be beneficial for their survival, e.g., in encapsulation devices, and thus be superior to temporal inhibition of apoptosis by a small compound apoptosis inhibitor which is currently being tested in a phase I/II clinical trial (NCT01653899). We thus generated transgenic pigs expressing XIAP under the control of the porcine INS promoter in the pancreatic beta-cells. Islets from these pigs are currently being tested vs. WT islets in staurosporine-induced apoptosis assays and transplantation experiments with reduced islet numbers.

Efficient revascularization of transplanted islets is an essential component to support their engraftment as well as longterm survival and function. Recent attempts focus on preconditioning of the transplantation site, e.g., by creating a prevascularized, subcutaneous pouch for islet transplantation, which supported islet graft survival and function in STZinduced diabetic mice, resulting in diabetes reversal in $>90 \%$ of the treated animals [82]. Another possibility is to stimulate revascularization of transplanted islets by local application or expression of angiogenic factors. After transplantation under the kidney capsule of syngeneic diabetic mice, transgenic mouse islets expressing vascular endothelial growth factor A (VEGFA) under the control of a rat insulin promoter showed enhanced microvascular density and functional blood flow to the graft compared with WT islets [83]. A similar positive effect of increased VEGFA production on graft angiogenesis and islet revascularization was observed after adenoviral transduction of mouse islets with a VEFGA expression cassette [84]. We have adapted a recently established inducible transgene expression system based on the binary Tet-On system in pigs [85] for beta-cell specific inducible expression of VEGFA and will test the revascularization capacity of islets from such donor pigs vs. WT islets after transplantation into the anterior chamber of the mouse eye $[86,87]$ and after transplantation under the kidney capsule of diabetic mice [69॰].

Recently, a transgenic strategy to improve the insulin secretion capacity of porcine islets, which is markedly lower compared to human and NHP islets [88], was suggested. Mourad et al. [89] demonstrated that glucagon-like peptide 1 (GLP1) and M3 muscarinic acetylcholine receptors expressed from viral vectors in the beta-cells act synergistically to enhance insulin secretion by adult porcine islets and NICCs. Attempts to test this concept in vivo in transgenic pigs were announced.

For a considerable period of time, PERVs have been seen as a major hurdle in xenotransplantation [90] but more recent data gained from studies in primates or human beings that received porcine grafts did not provide any evidence of PERV transmission so far. Nonetheless, the endogenous nature of these viruses in xenografts represents a permanent scare and, thus, different strategies, such as choosing donor animals with low PERV expression or knocking down PERV expression by transgenic approaches, have been presented to minimize this hazardous potential [91]. Due to the multiple PERV loci in the porcine genome, a complete removal of PERV from donor pigs seemed not to be a realistic approach, but very recently, all PERV integrants in a porcine cell line have been disrupted in a single attempt of gene editing with CRISPR/Cas9. This was achieved by using guide RNAs targeting the retroviral pol gene which is highly homologous in PERV A, B, and C [92•], opening the perspective of PERVfree donor pigs.

The aims of genetic modifications of islet donor pigs and their relevance for free vs. encapsulated islet transplantation are shown in Fig. 1.

In summary, only few systematic efficacy studies of genetically modified porcine islets in NHP have been performed, with a focus on preventing complement mediated processes such as IBMIR. Their results suggest that genetic modification of the donor pigs will be important, perhaps essential, for the success of clinical porcine islet xenotransplantation. High costs and ethical constraints of NHP studies limit the number 
of recipients for testing genetically (multi-)modified pig islets. Thus, there is a need for a hierarchical screening pipeline of in vitro assays, improved "humanized" mouse models with all subsets of human lymphoid and myeloid cells (e.g., [93]) and large diabetic animal models such as the C94Y insulin mutant "Akita" pig model [94•] for efficacy testing.

\section{Conclusion}

Xenotransplantation of porcine islets is currently the most advanced alternative to pancreas or islet allotransplantation and will probably remain a relevant option for specific patient groups even if stem cell-derived beta-cells prove to be clinically effective. Genetic modification of the islet donor pigs will result in optimized islet grafts which are protected against immune rejection and are improved in viability and function. The latter is not only relevant for transplantation of free islets but also for various encapsulation strategies.

Acknowledgments The authors acknowledge support by the Deutsche Forschungsgemeinschaft (Transregional Collaborative Research Center 127 "Biology of xenogeneic cell, tissue and organ transplantation-from bench to bedside"). N.K. and E.W. are members of EU COST Action BM1308 "Sharing Advances on Large Animal Models-SALAAM."

\section{Compliance with Ethical Standards}

Conflict of Interest Nikolai Klymiuk, Barbara Ludwig, Jochen Seissler, Bruno Reichart, and Eckhard Wolf declare that they have no conflict of interest.

Human and Animal Rights and Informed Consent This article does not contain any studies with human or animal subjects performed by any of the authors.

\section{References}

Papers of particular interest, published recently, have been highlighted as:

- Of importance

1. Atkinson MA, Eisenbarth GS, Michels AW. Type 1 diabetes. Lancet. 2014;383:69-82.

2. Diaz-Valencia PA, Bougneres P, Valleron AJ. Global epidemiology of type 1 diabetes in young adults and adults: a systematic review. BMC Public Health. 2015;15:255.

3. American Diabetes Association. Approaches to glycemic treatment. Sec. 7. In standards of medical care in diabetes - 2015. Diabetes Care. 2015;38 Suppl 1:S41-8.

4. Yeh HC, Brown TT, Maruthur N, et al. Comparative effectiveness and safety of methods of insulin delivery and glucose monitoring for diabetes mellitus: a systematic review and meta-analysis. Ann Intern Med. 2012;157:336-47.
5. Farrington $\mathrm{C}$. The artificial pancreas: challenges and opportunities. Lancet Diabetes Endocrinol. 2015;3:937.

6. Choudhary P, Rickels MR, Senior PA, et al. Evidence-informed clinical practice recommendations for treatment of type 1 diabetes complicated by problematic hypoglycemia. Diabetes Care. 2015;38:1016-29.

7. Forlenza GP, Buckingham B, Maahs DM. Progress in diabetes technology: developments in insulin pumps, continuous glucose monitors, and progress towards the artificial pancreas. J Pediatr. 2016;169:13-20.

8. Reichart B, Niemann H, Chavakis T, et al. Xenotransplantation of porcine islet cells as a potential option for the treatment of type 1 diabetes in the future. Horm Metab Res. 2015;47:31-5.

9. Niclauss N, Morel P, Berney T. Has the gap between pancreas and islet transplantation closed? Transplantation. 2014;98:593-9.

10. Ludwig B, Ludwig S, Steffen A, Saeger HD, Bornstein SR. Islet versus pancreas transplantation in type 1 diabetes: competitive or complementary? Curr Diab Rep. 2010;10:506-11.

11. Barton FB, Rickels MR, Alejandro R, et al. Improvement in outcomes of clinical islet transplantation: 1999-2010. Diabetes Care. 2012;35:1436-45.

12. Hering BJ, Bellin MD. Transplantation: Sustained benefits of islet transplants for T1DM. Nat Rev Endocrinol. 2015;11:572-4.

13. Pagliuca FW, Melton DA. How to make a functional beta-cell. Development. 2013;140:2472-83.

14. Ariyachet C, Tovaglieri A, Xiang G, et al. Reprogrammed stomach tissue as a renewable source of functional $\beta$ cells for blood glucose regulation. Cell Stem Cell. 2016;18:1-12. This paper shows that cells of the antral stomach region of mice can be reprogrammed to a beta-cell fate and that bioengineered stomach organoid gafts with these cells can rescue hyperglycemia in diabetic mice.

15. Blum B, Hrvatin SS, Schuetz C, Bonal C, Rezania A, Melton DA. Functional beta-cell maturation is marked by an increased glucose threshold and by expression of urocortin 3. Nat Biotechnol. 2012;30:261-4.

16. Pagliuca FW, Millman JR, Gurtler M, et al. Generation of functional human pancreatic beta cells in vitro. Cell. 2014;159:428-39. This paper reports a scalable differentiation protocol for human pluripotent stem cells that can generate glucose-responsive betacells, which secrete human insulin and are able to ameliorate hyperglycemia after transplantation into diabetic mice.

17. Rezania A, Bruin JE, Riedel MJ, et al. Maturation of human embryonic stem cell-derived pancreatic progenitors into functional islets capable of treating pre-existing diabetes in mice. Diabetes. 2012;61:2016-29.

18. Rezania A, Bruin JE, Xu J, et al. Enrichment of human embryonic stem cell-derived NKX6.1-expressing pancreatic progenitor cells accelerates the maturation of insulin-secreting cells in vivo. Stem Cells. 2013;31:2432-42.

19. Vegas AJ, Veiseh O, Gurtler M, et al. Long-term glycemic control using polymer-encapsulated human stem cell-derived beta cells in immune-competent mice. Nat Med. 2016. doi:10.1038/nm.4030. This paper reports the first long-term correction of diabetes in immunocompetent mice by transplantation of human pluripotent stem cell-derived beta-cells encapsulated with alginate derivatives.

20. Vegas AJ, Veiseh O, Doloff JC, et al. Combinatorial hydrogel library enables identification of materials that mitigate the foreign body response in primates. Nat Biotechnol. 2016. doi:10.1038/ nbt.3462. This study used a combinatorial approach for covalent chemical modifications of alginate and tested their biocompatibility in non-human primates.

21. Agulnick AD, Ambruzs DM, Moorman MA, et al. Insulinproducing endocrine cells differentiated in vitro from human embryonic stem cells function in macroencapsulation devices in vivo. Stem Cells Transl Med. 2015;4:1214-22. 
22. Kobayashi T, Yamaguchi T, Hamanaka S, et al. Generation of rat pancreas in mouse by interspecific blastocyst injection of pluripotent stem cells. Cell. 2010;142:787-99.

23. Matsunari H, Nagashima H, Watanabe M, et al. Blastocyst complementation generates exogenic pancreas in vivo in apancreatic cloned pigs. Proc Natl Acad Sci U S A. 2013;110:4557-62. This paper reports the generation of allogeneic pig pancreas by complementation of pancreatogenesis-disabled pig blastocysts with intact embryonic blastomeres from a different genetic background

24. Masaki H, Kato-Itoh M, Umino A, et al. Interspecific in vitro assay for the chimera-forming ability of human pluripotent stem cells. Development. 2015;142:3222-30.

25. Hermeren G. Ethical considerations in chimera research. Development. 2015;142:3-5.

26. Kobayashi T, Kato-Itoh M, Nakauchi H. Targeted organ generation using Mixl1-inducible mouse pluripotent stem cells in blastocyst complementation. Stem Cells Dev. 2015;24:182-9.

27. Wu J, Izpisua Belmonte JC. Dynamic pluripotent stem cell states and their applications. Cell Stem Cell. 2015;17:509-25. Excellent review of different strategies of interspecies chimeric complementation.

28. Takebe T, Enomura M, Yoshizawa E, et al. Vascularized and complex organ buds from diverse tissues via mesenchymal cell-driven condensation. Cell Stem Cell. 2015;16:556-65.

29. Yokote S, Matsunari H, Iwai S, et al. Urine excretion strategy for stem cell-generated embryonic kidneys. Proc Natl Acad Sci U S A. 2015;112:12980-5.

30. Kemter E, Wolf E. Pigs pave a way to de novo formation of functional human kidneys. Proc Natl Acad Sci U S A. 2015;112:12905-6.

31. Lee K, Kwon DN, Ezashi T, et al. Engraftment of human iPS cells and allogeneic porcine cells into pigs with inactivated RAG2 and accompanying severe combined immunodeficiency. Proc Natl Acad Sci U S A. 2014;111:7260-5.

32. Dufrane D, Gianello P. Macro- or microencapsulation of pig islets to cure type 1 diabetes. World J Gastroenterol. 2012;18:6885-93.

33. Nagaraju S, Bottino R, Wijkstrom M, Trucco M, Cooper DK. Islet xenotransplantation: what is the optimal age of the islet-source pig? Xenotransplantation. 2015;22:7-19.

34. Cardona K, Korbutt GS, Milas Z, et al. Long-term survival of neonatal porcine islets in nonhuman primates by targeting costimulation pathways. Nat Med. 2006;12:304-6.

35. Hering BJ, Wijkstrom M, Graham ML, et al. Prolonged diabetes reversal after intraportal xenotransplantation of wild-type porcine islets in immunosuppressed nonhuman primates. Nat Med. 2006;12:301-3.

36. Thompson P, Badell IR, Lowe M, et al. Alternative immunomodulatory strategies for xenotransplantation: CD40/154 pathwaysparing regimens promote xenograft survival. Am J Transplant. 2012;12:1765-75.

37. Shin JS, Kim JM, Kim JS, et al. Long-term control of diabetes in immunosuppressed nonhuman primates (NHP) by the transplantation of adult porcine islets. Am J Transplant. 2015;15:2837-50. This paper describes the longest survival and function of wildtype pig islets in diabetic rhesus macaques with immunosuppression

38. Kawai T, Andrews D, Colvin RB, Sachs DH, Cosimi AB. Thromboembolic complications after treatment with monoclonal antibody against CD40 ligand. Nat Med. 2000;6:114.

39. Ludwig B, Ludwig S. Transplantable bioartificial pancreas devices: current status and future prospects. Langenbeck's Arch Surg. 2015;400:531-40.

40. Robles L, Storrs R, Lamb M, Alexander M, Lakey JR. Current status of islet encapsulation. Cell Transplant. 2014;23:1321-48.

41. Dufrane D, Goebbels RM, Saliez A, Guiot Y, Gianello P. Six-month survival of microencapsulated pig islets and alginate biocompatibility in primates: proof of concept. Transplantation. 2006;81:1345-53.

42. Casu A, Bottino R, Balamurugan AN, et al. Metabolic aspects of pig-to-monkey (Macaca fascicularis) islet transplantation: implications for translation into clinical practice. Diabetologia. 2008;51: $120-9$

43. Dufrane D, Steenberghe M, Goebbels RM, Saliez A, Guiot Y, Gianello P. The influence of implantation site on the biocompatibility and survival of alginate encapsulated pig islets in rats. Biomaterials. 2006;27:3201-8.

44. Yang HK, Ham DS, Park HS, et al. Long-term efficacy and biocompatibility of encapsulated islet transplantation with chitosancoated alginate capsules in mice and canine models of diabetes. Transplantation. 2016;100:334-43.

45. Matsumoto S, Tan P, Baker J, et al. Clinical porcine islet xenotransplantation under comprehensive regulation. Transplant Proc. 2014;46:1992-5.

46. Wynyard S, Nathu D, Garkavenko O, Denner J, Elliott R. Microbiological safety of the first clinical pig islet xenotransplantation trial in New Zealand. Xenotransplantation. 2014;21:309-23.

47. Hill RS, Cruise GM, Hager SR, et al. Immunoisolation of adult porcine islets for the treatment of diabetes mellitus. The use of photopolymerizable polyethylene glycol in the conformal coating of mass-isolated porcine islets. Ann N Y Acad Sci. 1997;831:33243.

48. Tomei AA, Manzoli V, Fraker CA, et al. Device design and materials optimization of conformal coating for islets of Langerhans. Proc Natl Acad Sci U S A. 2014;111:10514-9. This paper describes novel approaches and instruments for conformal coating of pancreatic islets.

49. Dufrane D, Goebbels RM, Gianello P. Alginate macroencapsulation of pig islets allows correction of streptozotocin-induced diabetes in primates up to 6 months without immunosuppression. Transplantation. 2010;90:1054-62.

50. Neufeld T, Ludwig B, Barkai U, et al. The efficacy of an immunoisolating membrane system for islet xenotransplantation in minipigs. PLoS One. 2013;8, e70150.

51. Ludwig B, Reichel A, Steffen A, et al. Transplantation of human islets without immunosuppression. Proc Natl Acad Sci U S A. 2013;110:19054-8. This paper reports long-term survival of allogeneic human islets in an oxygenated chamber system transplanted in a type 1 diabetic patient without immunosuppression.

52. Ludwig B, Rotem A, Schmid J, et al. Improvement of islet function in a bioartificial pancreas by enhanced oxygen supply and growth hormone releasing hormone agonist. Proc Natl Acad Sci U S A. 2012;109:5022-7.

53. Ludwig B, Ludwig S, Steffen A, et al. Preclinical studies on porcine islet macroencapsulation in non-human primates. Xenotransplantation. 2015;22 Suppl 1:S19-20.

54. Bottino R, Trucco M. Use of genetically-engineered pig donors in islet transplantation. World J Transplant. 2015;5:243-50.

55. van der Windt DJ, Marigliano M, He J, et al. Early islet damage after direct exposure of pig islets to blood: has humoral immunity been underestimated? Cell Transplant. 2012;21:1791-802.

56. Rayat GR, Rajotte RV, Hering BJ, Binette TM, Korbutt GS. In vitro and in vivo expression of Galalpha-(1,3)Gal on porcine islet cells is age dependent. J Endocrinol. 2003;177:127-35.

57. van der Windt DJ, Bottino R, Casu A, et al. Long-term controlled normoglycemia in diabetic non-human primates after transplantation with hCD46 transgenic porcine islets. Am J Transplant. 2009;9:2716-26.

58. Thompson P, Badell IR, Lowe M, et al. Islet xenotransplantation using gal-deficient neonatal donors improves engraftment and function. Am J Transplant. 2011;11:2593-602. 
59. Hawthorne WJ, Salvaris EJ, Phillips P, et al. Control of IBMIR in neonatal porcine islet xenotransplantation in baboons. Am J Transplant. 2014;14:1300-9.

60. Martin BM, Samy KP, Lowe MC, et al. Dual islet transplantation modeling of the instant blood-mediated inflammatory reaction. Am J Transplant. 2015;15:1241-52. This paper describes an elegant transplantation model in rhesus macaques, where islets from two different sources are infused into distictly vascularized liver lobes and can thus be tested in the same recipient.

61. Shapiro AM. Islet transplantation in type 1 diabetes: ongoing challenges, refined procedures, and long-term outcome. Rev Diabet Stud. 2012;9:385-406.

62. Bertuzzi F, Marzorati S, Maffi P, et al. Tissue factor and CCL2/monocyte chemoattractant protein-1 released by human islets affect islet engraftment in type 1 diabetic recipients. J Clin Endocrinol Metab. 2004;89:5724-8.

63. Lee I, Wang L, Wells AD, et al. Blocking the monocyte chemoattractant protein-1/CCR2 chemokine pathway induces permanent survival of islet allografts through a programmed death-1 ligand-1-dependent mechanism. J Immunol. 2003;171:6929-35.

64. Moberg L, Johansson H, Lukinius A, et al. Production of tissue factor by pancreatic islet cells as a trigger of detrimental thrombotic reactions in clinical islet transplantation. Lancet. 2002;360:2039 45.

65. Kourtzelis I, Magnusson PU, Kotlabova K, Lambris JD, Chavakis T. Regulation of instant blood mediated inflammatory reaction (IBMIR) in pancreatic islet xeno-transplantation: points for therapeutic interventions. Adv Exp Med Biol. 2015;865:171-88.

66. Lu B, Rutledge BJ, Gu L, et al. Abnormalities in monocyte recruitment and cytokine expression in monocyte chemoattractant protein 1-deficient mice. J Exp Med. 1998;187:601-8.

67. Wood JP, Ellery PE, Maroney SA, Mast AE. Biology of tissue factor pathway inhibitor. Blood. 2014;123:2934-43.

68. Soderlund J, Wennberg L, Castanos-Velez E, et al. Fetal porcine islet-like cell clusters transplanted to cynomolgus monkeys: an immunohistochemical study. Transplantation. 1999;67:784-91.

69. Klymiuk N, van Buerck L, Bahr A, et al. Xenografted islet cell clusters from INSLEA29Y transgenic pigs rescue diabetes and prevent immune rejection in humanized mice. Diabetes. 2012;61: 1527-32. This paper shows that local expression of LEA29Y in transgenic pig islet grafts prevents their rejection in mice with human immune cells.

70. Wolf-van Buerck L, Schuster M, Baehr A, et al. Engraftment and reversal of diabetes after intramuscular transplantation of neonatal porcine islet-like clusters. Xenotransplantation. 2015;22:443-50.

71. Plotzki E, Wolf-van Buerck L, Knauf Y, et al. Virus safety of islet cell transplantation from transgenic pigs to marmosets. Virus Res. 2015;204:95-102.

72. Plege A, Borns K, Baars W, Schwinzer R. Suppression of human Tcell activation and expansion of regulatory $\mathrm{T}$ cells by pig cells overexpressing PD-ligands. Transplantation. 2009;87:975-82.

73. Plege-Fleck A, Lieke T, Romermann D, et al. Pig to rat cell transplantation: reduced cellular and antibody responses to xenografts overexpressing PD-L1. Xenotransplantation. 2014;21:533-42.

74. Yi S, Feng X, Hawthorne WJ, Patel AT, Walters SN, O'Connell PJ. $\mathrm{CD} 4+\mathrm{T}$ cells initiate pancreatic islet xenograft rejection via an interferon-gamma-dependent recruitment of macrophages and natural killer cells. Transplantation. 2002;73:437-46.

75. Weiss EH, Lilienfeld BG, Muller S, et al. HLA-E/human beta2microglobulin transgenic pigs: protection against xenogeneic human anti-pig natural killer cell cytotoxicity. Transplantation. $2009 ; 87: 35-43$.
76. Byrne GW, McGregor CG, Breimer ME. Recent investigations into pig antigen and anti-pig antibody expression. Int J Surg. 2015;23: 223-8.

77. Bottino $\mathrm{R}$, Hara $\mathrm{H}$, Iwase $\mathrm{H}$, et al. In vitro exposure of isolated porcine islets from Neu5Gc-knockout/GTknockout/hCD46 transgenic donors to human blood/plasma. Xenotransplantation. 2015;22 Suppl 1:S65-6.

78. Park CG, Bottino R, Hawthorne WJ. Current status of islet xenotransplantation. Int J Surg. 2015;23:261-6.

79. Wijkstrom M, Bottino R, Iwase H, et al. Glucose metabolism in pigs expressing human genes under an insulin promoter. Xenotransplantation. 2015;22:70-9.

80. Bottino R, Wijkstrom M, van der Windt DJ, et al. Pig-to-monkey islet xenotransplantation using multi-transgenic pigs. Am J Transplant. 2014;14:2275-87.

81. Emamaullee JA, Rajotte RV, Liston P, et al. XIAP overexpression in human islets prevents early posttransplant apoptosis and reduces the islet mass needed to treat diabetes. Diabetes. 2005;54:2541-8.

82. Pepper AR, Gala-Lopez B, Pawlick R, Merani S, Kin T, Shapiro AMJ. A prevascularized subcutaneous device-less site for islet and cellular transplantation. Nat Biotechnol. 2015;33:518-23.

83. Lai Y, Schneider D, Kidszun A, et al. Vascular endothelial growth factor increases functional beta-cell mass by improvement of angiogenesis of isolated human and murine pancreatic islets. Transplantation. 2005;79:1530-6.

84. Zhang N, Richter A, Suriawinata J, et al. Elevated vascular endothelial growth factor production in islets improves islet graft vascularization. Diabetes. 2004;53:963-70.

85. Klymiuk N, Bocker W, Schonitzer V, et al. First inducible transgene expression in porcine large animal models. FASEB J. 2012;26: 1086-99.

86. Speier $\mathrm{S}$, Nyqvist $\mathrm{D}$, Cabrera $\mathrm{O}$, et al. Noninvasive in vivo imaging of pancreatic islet cell biology. Nat Med. 2008;14:574-8.

87. Speier S, Nyqvist D, Kohler M, Caicedo A, Leibiger IB, Berggren PO. Noninvasive high-resolution in vivo imaging of cell biology in the anterior chamber of the mouse eye. Nat Protoc. 2008;3:127886.

88. Mueller KR, Balamurugan AN, Cline GW, et al. Differences in glucose-stimulated insulin secretion in vitro of islets from human, nonhuman primate, and porcine origin. Xenotransplantation. 2013;20:75-81.

89. Mourad N, Xhema D, Perota A, Galli C, Gianello P. Beta-cellspecific expression of glucagon-like peptide 1 (GLP-1) and activated muscarinic receptor (M3R) improves pig islet secretory function. Xenotransplantation. 2015;22 Suppl 1:S176.

90. Denner J, Schuurman HJ, Patience C. The International Xenotransplantation Association consensus statement on conditions for undertaking clinical trials of porcine islet products in type 1 diabetes-chapter 5: Strategies to prevent transmission of porcine endogenous retroviruses. Xenotransplantation. 2009;16:239-48.

91. Denner J, Mueller NJ. Preventing transfer of infectious agents. Int J Surg. 2015;23:306-11.

92. Yang L, Guell M, Niu D, et al. Genome-wide inactivation of porcine endogenous retroviruses (PERVs). Science (New York, NY). 2015;350:1101-4. This study used the CRISPR/Cas system to mutate all copies of the PERV pol gene in a porcine cell line.

93. Cosgun KN, Rahmig S, Mende N, et al. Kit regulates HSC engraftment across the human-mouse species barrier. Cell Stem Cell. 2014;15:227-38.

94. Renner S, Braun-Reichhart C, Blutke A, et al. Permanent neonatal diabetes in INS(C94Y) transgenic pigs. Diabetes. 2013;62:150511. This paper reports the first genetically diabetic pig model. 